\title{
Tracheal and carinal resections in the treatment of non-small cell lung cancer: a trend to a minimally invasive approach
}

\author{
William Guido-Guerrero ${ }^{1}$, Albert Bolaños-Cubillo ${ }^{1}$, Alejandro García-Pérez ${ }^{2}$, Diego González-Rivas $^{2,3}$ \\ ${ }^{1}$ Department of Thoracic Surgery, Rafael Angel Calderón Guardia Hospital, San José, Costa Rica; ${ }^{2}$ Department of Thoracic Surgery, Coruña \\ University Hospital and Minimally Invasive Thoracic Surgery Unit (UCTMI), Coruña, Spain; ${ }^{3}$ Department of Thoracic Surgery, Shanghai \\ Pulmonary Hospital, Tongji University School of Medicine, Shanghai, China \\ Contributions: (I) Conception and design: W Guido Guerrero, D González-Rivas; (II) Administrative support: W Guido Guerrero, D González- \\ Rivas; (III) Provision of study materials or patients: All authors; (IV) Data collection and assembly: All authors; (V) Data analysis and interpretation: \\ All authors; (VI) Manuscript writing: All authors; (VII) Final approval of manuscript: All authors. \\ Correspondence to: William Guido-Guerrero. Department of Thoracic Surgery, Rafael Angel Calderón Guardia Hospital, San José, Costa Rica. \\ Email: guido.william@gmail.com.
}

\begin{abstract}
Surgical treatment for non-small cell lung cancer (NSCLC) involving trachea and carina is one of the most infrequent and challenging procedures for a thoracic surgeon, requiring careful preoperative assessment, high level management of the airway, advanced surgical technique and appropriate postoperative care. Carinal and tracheal resections with lung sparing, and carinal sleeve pneumonectomy are part of these complex techniques, with a variable rate of morbidity and mortality. To prevent complications, the most important aspect of the technique is for reconstruction to be performed without tension and with a good blood supply. If a complication is suspected, it must be diagnosed at an early stage, and the problem must be handled aggressively to avoid further progression. The majority of cases are performed with an open approach, but under the care of surgeons and anesthesiologists experienced in minimally invasive thoracic procedures, with advanced airway management skills, in high volume referral centers, these procedures can be performed safely with video-assisted thoracic surgery to obtain good postoperative results. In recent years, with the rapid development of uniportal video assisted thoracic surgery (VATS), this approach resulted in shorter postoperative stays, less postoperative pain and faster recovery after surgery, maintaining oncological principles, becoming an excellent alternative for these difficult cases. This article aims to review the specific considerations that involve this kind of tumors, surgical technique and anesthesiology options, and the recent shift to a minimally invasive approach.
\end{abstract}

Keywords: Tracheal; carinal; minimally invasive thoracic surgery; uniportal

Received: 03 May 2020; Accepted: 11 June 2020; Published: 25 August 2021.

doi: $10.21037 /$ ccts-20-95

View this article at: http://dx.doi.org/10.21037/ccts-20-95

\section{Introduction}

Tumors involving the carina or distal trachea are usually advanced stages of non-small cell lung cancer (NSCLC), commonly accompanied by mediastinal lymph node involvement. A large proportion of this cases are usually treated with chemo and radiotherapy. Despite this, there are some cases that are amenable to a curative resection and, because of the circumstances involving this anatomical location are among the most challenging cases for thoracic surgeons.

Surgical treatment on NSCLC is evolving every day. Boundaries are continuously pushed to offer a curative treatment, not only for early stages of lung cancer, but for more advanced cases that require complex anatomical resections and reconstructions. Tracheal and carinal resections are one of the most difficult procedures for the treatment of NSCLC, defying thoracic surgeons and anesthesiologists alike. Careful preoperative assessment 
must be carried out between both medical professionals due to specific considerations involving airway management during this type of surgery.

Early reports of carinal reconstruction date back to 1950 s and 60s, when teams lead by Abbot and Barclay showed that this type of procedures where possible, despite being associated with considerable morbi-mortality $(1,2)$. As a result of those first reports, surgeons seldom performed them. Advances in airway management, postoperative care and surgical technology has resulted in considerably lower complications following these procedures. Although, more than five decades have gone by since the first cases were treated and because of the technical complexity, the majority of teams performing these types of surgeries do it with an open approach.

Minimally invasive thoracic surgery has taken giant leaps in the last decade with the evolution of video assisted thoracic surgery (VATS) (3), and it has allowed experienced groups around the world to successfully perform this procedures by VATS, thus starting a discussion in which, the debate involves if the advantage of minimally invasive approach surpasses the technical demands and risks of performing these type of surgeries with a minimally invasive approach. More time and experience will be needed to answer this question, but for now the major limitation seems to be the experience of the surgical team with VATS. Reports available have shown that in the hands of highly experience VATS surgeons and anesthesiologists this approach is a feasible option for patients afflicted with this advanced stage lung cancers (4).

This article aims to review the specific considerations that involve this kind of tumors, surgical and anesthesiology options involving the approach and the recent shift to a minimally invasive approach.

\section{What are the scenarios in which these types of procedures are considered?}

The usual scenario is a patient with an upper lobe lung cancer that involves the main bronchus at less than $1 \mathrm{~cm}$ from the carina or a primary airway tumor located in the distal trachea, main bronchus or carina. Because of its location, mediastinal lymph node involvement is not rare and must be established before selecting the patient for surgery.

The most important aspect to be always kept in mind is that the oncologic principles must always be respected in order to appropriately select the patients for surgery.
Case selection is very important and must involve diverse factors, beginning with tumor localization and staging, patient condition and comorbidities and anatomical considerations. Once these aspects are clear, an adequate treatment plan can be tailored to each case (5).

\section{Localization and staging}

The majority of tracheobronchial tumors involving the carina or distal trachea are usually malignant and the predominant histologic types are squamous cell carcinoma (SCC), carcinoid tumors, mucoepidermoid carcinoma, and adenoid cystic carcinoma (5).

With regards to tumor location and extension, is important to keep in mind that resectability of the lesion is also determined by the extent of the airway involvement, since lesions that require more than $4 \mathrm{~cm}$ of distal trachea for pure tracheal resections, or $2 \mathrm{~cm}$ of distal trachea and $1.5 \mathrm{~cm}$ of proximal bronchus for carinal resections are usually not amenable to reconstruction $(6,7)$.

The best option for identifying endoluminal airway involvement is with bronchoscopy, as it can clearly define macroscopical extension proximally and distally in the airway, allowing for a more precise surgical planning (8). In the past, some authors have even suggested taking proximal and distal biopsies from the lesion to confirm if a negative margin tension free anastomosis will be possible (9).

Involvement of N2 is of special consideration. Evaluation of the mediastinal lymph nodes prior to surgery is better done by endobronchial ultrasound (EBUS), as it avoids scarring and fibrosis around the trachea, adding more difficulty to an already complex tracheal and carinal mobilization during the definitive surgery (10). For this reason, mediastinoscopy is usually reserved to be performed at the same surgical time as the definitive surgery to rule out persistent $\mathrm{N} 2$ disease after neoadjuvant therapy and can even help facilitate the dissection that will be necessary to mobilize the trachea for the anastomosis.

Although N2 confirmation prior to surgery usually determines if the patient is a candidate for definitive chemoradiotherapy instead of surgery, there are some groups currently performing surgery for these cases with good results (11), especially with single level N2 disease (12), so it should not be considered a contraindication for surgery. It is important to interpret these results with care and in the context of high-volume centers, since it is clear that neoadjuvant therapy is a risk factor for intraoperative complications and anastomotic failure. Thoracic surgical 
teams that decide to operate in N2 cases that need tracheobronchial reconstructions must be highly experienced and already have experience with this kind of resections without mediastinal lymph node involvement $(13,14)$.

\section{Patient condition and comorbidities}

Since airway reconstruction procedures involve extensive dissection, hilar manipulation and, sometimes, extensive parenchymal resections (sleeve pneumonectomy) they can be accompanied by postoperative complications, such as pneumonia, acute respiratory distress syndrome (ARDS) and arrhythmia (15). Because of this, the patient must be fit to tolerate this type of procedures an exhaustive preoperative evaluation is mandatory to assess the overall condition of the patient.

Medical history detailing prior or current smoking history, medications taken by the patient, complete blood test, cardiac and pulmonary function tests, including 6-minute walking tests, ventilation/perfusion scans (especially important when pneumonectomy is a possibility), echocardiogram and oxygen consumption tests should be obtained (16). Patients with chronic use of steroids and prior tracheal irradiation with more than $50 \mathrm{~Gy}(8)$ are more prone to anastomotic complications due to impaired healing $(7,17)$. Steroids should be avoided in the perioperative period if possible, to diminish the risk of dehiscence and anastomotic failure.

A multidisciplinary team must assess all patients with lung cancer who are candidates for surgical resection (18). This team should include the thoracic surgeon, pulmonologist, anesthesiologist, oncologist, and cardiologist $(18,19)$. After surgery, patients who are potential candidates for curative surgical resection could have a variable functional impairment in cardiac and respiratory systems with potential severe complications- Guidelines recommend that these patients should undergo spirometry (FEV1) and diffusing capacity of carbon monoxide (DLCO), and should be calculated the predicted postoperative FEV1 and DLCO (18-20). More than $60 \%$ of postoperative predicted FEV1 and DLCO indicate a low risk of perioperative death and cardiopulmonary complications following resection, including pneumonectomy $(18,21)$.

\section{Special considerations in the perioperative management}

The type of procedure to be performed depends mainly on the anatomic location of the tumor and its extension.

After a careful preoperative evaluation and planning, the final approach to the patient in the operation room implies a close coordination with the anesthesiologist, with diverse transoperative airway management options available for the surgical procedure.

\section{Anesthesiologic considerations}

For pure distal tracheal resections a single lumen tube is placed distal to the lesion for ventilation before airway transection (8). Total intravenous anesthesia (TIVA) is a good choice for tracheal resections, since it provides good relaxation, facilitating a steady field during dissection and eliminates airway reflexes. The effect is rapidly cleared so it allows extubation quickly after surgery is completed (17). After the airway is transected, the distal end of the trachea is intubated with sterile endotracheal tube connected to sterile tubing that also passes to the anesthesiologist (cross-field ventilation) (6). The anastomosis can be done with the tube in place, setting the sutures around it. However, if additional exposure is needed, the tube can be removed and parts of the anastomosis can be done during shorts periods of apnea (22). Another option is to use high frequency jet ventilation (HFJV). This option provides adequate oxygenation and only minimal interference in the surgical field. It can be advanced by the anesthesiologist and placed in the distal part with the help of the surgeon in the operative field (23).

For carinal resections, double-lumen endotracheal tubes are usually used for lung isolation during the beginning of the procedure (12), although some groups also advocate the use of single lumen tubes that are advanced to the main-stem bronchus $(7,10)$. In the same fashion as in tracheal resection, after airway is transected the opposite bronchus is intubated across the operative field or a HFJV catheter is advance from the endotracheal tube and into the selected bronchus to keep oxygenation during the anastomosis (9) (Figure 1). Although frequently advance through a supraglottic airway, the jet catheter can be placed also directly through across the field (22), but this could diminish the advantage offer by the device to interfere less in operative field.

In the majority of cases, cross field ventilation or HFJV provides good oxygenation and optimal exposure, but there are some circumstances in which extracorporeal membrane oxygenation (ECMO) may be needed.

Veno-venous ECMO is usually the preferred arrangement 


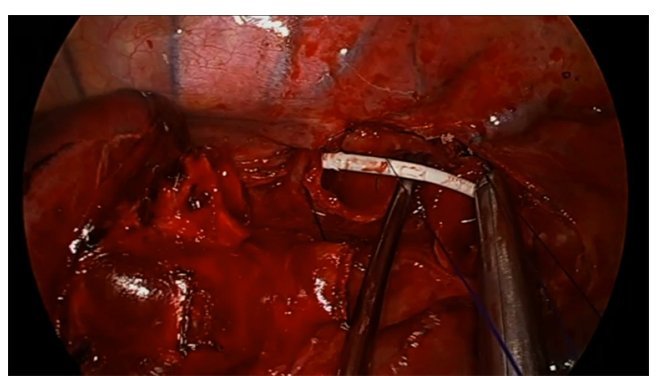

Figure 1 High frequency jet ventilation (HFJV) catheter advance into the left main bronchus for oxygenation during carinal reconstruction.

for airway surgery since it provides complete pulmonary support and has a diminished risk of vascular complications (24). The choice between a double cannulation vs a dual lumen single site cannulation setting will depend on the experience of the team performing the procedure and the availability of transesophageal echocardiography (TEE). Fluoroscopy can also be used to assess the correct positioning of the single lumen canula, but it could be more impractical and will require constant mobilization and interruption of the procedure to accommodate the equipment if misplacement occurs during surgery.

Veno-arterial ECMO is an option in cases where the patient has preexisting lung parenchymal diseases (16), hemodynamic support might be necessary during lung resection, considerable cardiac retraction could be necessary for exposure (25) or during intense emergency situations in which the hemodynamic conditions of the patient are already compromised (26).

Although considered a more invasive procedure, the use of ECMO does carry important exposure advantages, since oxygenation will be maintained without any ventilation system that interferes in the airway transection, manipulation and reconstruction, which could result in the procedure being completed within a shorter period of time (the anastomosis mainly).

More recently, the trend of less invasive procedures have also involved anesthesia with the advent of spontaneous ventilation-induced non intubated surgery (27). Avoiding mechanical ventilation avoids the side effects of general anesthesia and provides a more physiological status during the surgery (28). As with ECMO, this approach provides the advantage that there is no obstruction in the airway during the anastomosis and reconstruction. There are few reports in literature, especially by the Guangzhou Medical University group, reporting their experience (29). It is of upmost importance to emphasize that is a highly complex approach that requires a lot of coordination between the surgeon and the anesthesiologist and should only be perform when the team involved in the operation comprises experts in VATS airway reconstruction surgery and nonintubated anesthesia.

\section{Surgical options and the trend to a minimally invasive approach}

Often the vast majority of these cases are performed through an open surgery approach, not only because of the technical difficulty of the procedure itself but because is an infrequent scenario in the clinical practice of a thoracic surgeon. Hence, very few centers have experience with the surgical management of this tumors. It is very important that, in the event that one of these tumors is diagnosed, resectability should be evaluated by a multidisciplinary team in a tertiary center with experience of these anatomical locations (8).

Depending on the exact tumor location and its extension, there are different surgical techniques that provide an option for resection and subsequent reconstruction. The surgical options can be divided in:

(I) Lung sparring techniques

(i) Distal tracheal resection.

(ii) Carinal resection.

(II) Carinal and lung parenchymal resections

(i) Sleeve right pneumonectomy.

(ii) Sleeve left pneumonectomy.

(iii) Right upper lobectomy + carinal resection.

Right thoracotomy is usually the preferred access for distal tracheal resections, pure carinal reconstruction and right sided sleeve resections. For left sided sleeve carinal resections sternotomy it is usually the preferable access since the length of the left main bronchus (LMB) and aortic arch limit the exposure of the carina. A combination of sternotomy and left side VATS can be done in case of adhesions or hilar lymphadenopathy that complicates hilar dissection.

The key aspect of the procedure is that the reconstruction is made without tension and good blood supply. Release maneuvers like neck flexion, pretracheal plain development, hilar and ductus arteriosus release must be carried out to ensure a tension free anastomosis when needed. 
Table 1 Literature review of VATS carinal resection for NSCLC

\begin{tabular}{lccccl}
\hline Authors & Year & $\begin{array}{c}\text { Number of } \\
\text { cases }(\mathrm{n})\end{array}$ & $\begin{array}{c}\text { Number } \\
\text { of ports }\end{array}$ & $\begin{array}{c}\text { Perioperative } \\
\text { mortality }\end{array}$ & Major complications \\
\hline Nakanishi, et al. (31) & 2013 & 1 & 3 & None & None \\
Xu, et al. (33) & 2014 & 1 & 3 & None & NA \\
Lin, et al. (35) & 2015 & 1 & 5 & None & None \\
Lyscov, et al. (36) & 2016 & 2 & 1 & None & None \\
Gonzalez-Rivas, et al. (37) & 2016 & 3 & 1 & None & NA \\
Li, et al.* (32) & 2016 & 7 & $3-4$ & None & $42 \%$ pneumonia; 14\% pleural effusion \\
Qiu, et al. (38) & 2016 & 1 & 2 & None & None \\
Jiang, et al. (29) & 2018 & 6 & 3 & None & $25 \%$ pneumonia; 3\% bronchopleural fistula \\
\hline
\end{tabular}

*, for the purposes of this review the number of cases included on this table are only malignant disease patients. VATS, video-assisted thoracoscopic technique; NSCLC, non-small cell lung cancer; NA, not applicable.

\section{Minimally invasive approach}

Technology progress in VATS instrumentation, high definition cameras and the experience obtained in the last years by surgeons performing sleeve pulmonary resections, radical mediastinal lymphadenectomy and other kind of advance procedures have allowed some groups to successfully perform tracheal and carinal resection and reconstructions with a minimally invasive approach.

The first circumferential VATS tracheal resection and reconstruction was performed in 2005 (30) by Nakanishi et al. using a four ports arrangement. Eight years later, the first carinal reconstruction performed by VATS was reported by Nakanishi et al. in 2013. The case was performed with three ports conventional VATS and oxygenation was maintain with HFJV pass through the lumen of a bronchial blocker (31).

Since then, a few groups of highly experience VATS surgeons have reported their experience with a minimally invasive approach for this tumor locations (32-34) adding momentum to the technique. Reports of number of cases, minimally invasive approach and complications are available in a few reports in the literature (summarized in Table 1) (29,31-33,35-38). In more recent years the exponential growth experience in uniportal VATS have allowed for successful execution for these cases with good results $(39,40)$.

The rationale of doing such a complex VATS procedure is to offer this patients with advanced NSCLC the advantages of a minimally invasive approach such as a shorter postoperative stay, less postoperative pain and faster recovery (41).

\section{The rise of the uniportal approach}

From the different approaches in VATS surgery, uniportal VATS has experience in the last decade a rapid and continuous development in terms of its application for more advance cases and complex procedures $(3,4)$. The surgical procedure by itself is not different from open surgery and multiport VATS, so the oncological principles are not compromised. By using uniportal VATS the surgeon is able to complete the same procedures with less incisions and intercostal spaces affected in comparison with multiport VATS and has been associated with less postoperative pain (42).

Distal trachea and carinal resections are approach by uniportal VATS through the right hemithorax in a posteriorly place incision in the $3^{\text {rd }}$ or $4^{\text {th }}$ intercostal space, allowing a more comfortable access to the posterior mediastinum $(10,40)$. Instrumentation mimics the disposition in open surgery, with the camera (eyes) always in the upper part of the incision and carrying the dissection with bimanual instrumentation (Figure 2). Principles of dissection and exposure are the same as in open surgery, but visualization is usually better thanks to the proximity of the high definition cameras to the working field. Dissection is carried around the distal trachea and carina, the paratracheal space and tracheoesophageal groove are dissected respecting the lateral arterial perfusion (43). If the azygous vein interferes with the anastomosis it can be dissected apart or transected. A complete lymphadenectomy of station $4 \mathrm{R}$ and 7 can aid considerably in the exposure prior to airway transection and anastomosis. Circumference dissection is 


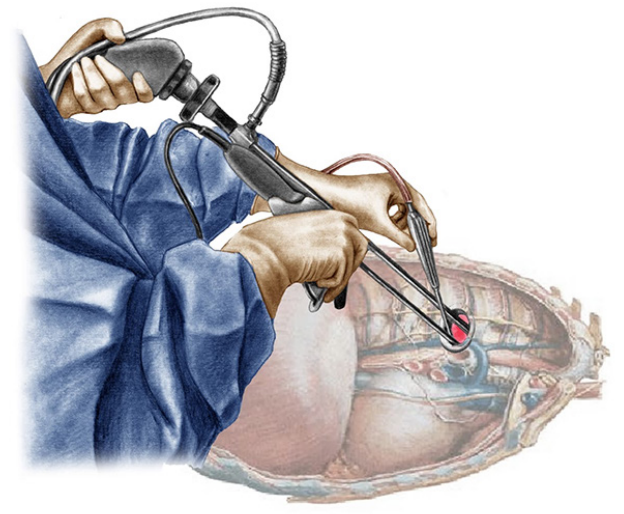

Figure 2 Incision placement and instrumentation for uniportal video assisted thoracic surgery (VATS) carinal dissection.

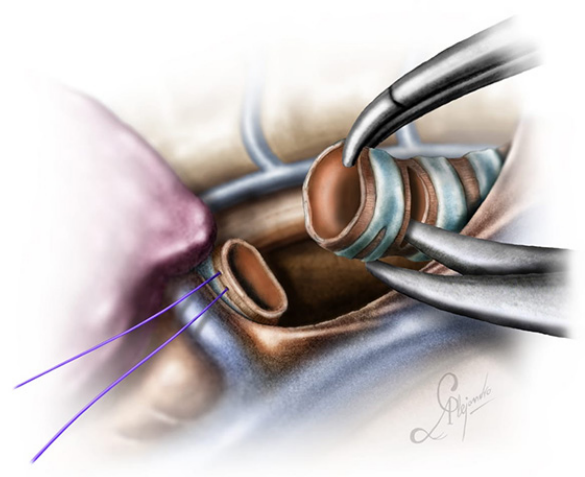

Figure 3 Traction and exposure during segmental resection of the distal trachea.

reserve to the segmental part of the trachea that will be resected. Isolation and traction of the trachea can be done with a vessel loop or a tape, and the transection is performed with scissors with the aid of instruments for exposure (Figure 3). A stay suture can be placed in distal airway for traction, which facilitates the anastomosis. The entire anastomosis is carried out with 3-0 PDS or polipropilene double needle suture with one running suture $(40,43)$.

Oxygenation and ventilation are maintained through guided advancement of a HFJV catheter inserted through the endotracheal tube and pass into the LMB. If HFJV is not available, cross field ventilation can be achieved with an additional $1-\mathrm{cm}$ incision through the surgical field $(27,37)$.

For carinal resections, the extent of the resection and type of reconstruction will depend on tumor involvement, sometimes even including the right upper lobe (Figure 4).

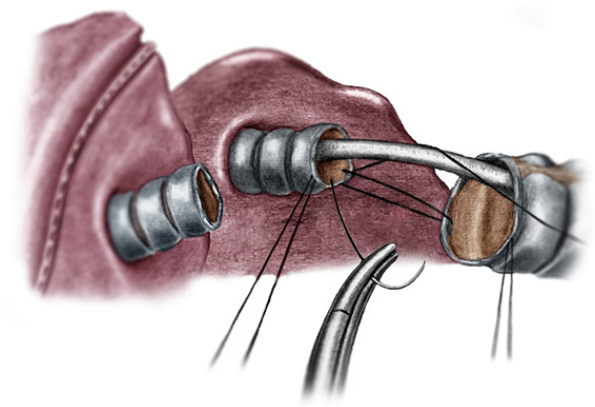

Figure 4 Carinal reconstruction after right upper lobectomy between the trachea, left main bronchus and intermediate bronchus.

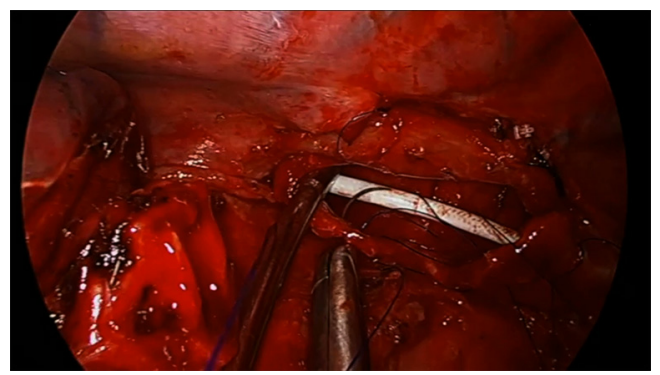

Figure 5 Running suture between the lateral part of the trachea and LMB. HFJV provides minor interference. LMB, left main bronchus; HFJV, high frequency jet ventilator.

The patient is also placed in left lateral decubitus and the recommended placement for the incision is the fourth intercostal space in the midaxillary line. The table is slightly tilted anteriorly to improve exposure of the posterior mediastinum (43). Dissection is then carried out through the paratracheal space and the trachea-esophageal groove. Conducting a complete lymph node dissection of station $2 \mathrm{R}, 4 \mathrm{R}$ and 7 facilitates airway isolation and transection afterwards. Dissection is carried around the LMB to facilitate exposure and posterior anastomosis. A suture can be placed in the LMB for retraction. The resection in the carina begins by cutting the distal trachea with scissors, revealing the endotracheal tube. The cut is then extended into the RMB and LMB until the carina is detached completely. The endotracheal tube is pulled back and HFJV is started after directing the catheter into the LMB, with minor interference during the anastomosis (Figure 5). The anastomosis is started with the lateral part of the LMB to the trachea (posterior side of the anastomosis). With a two 


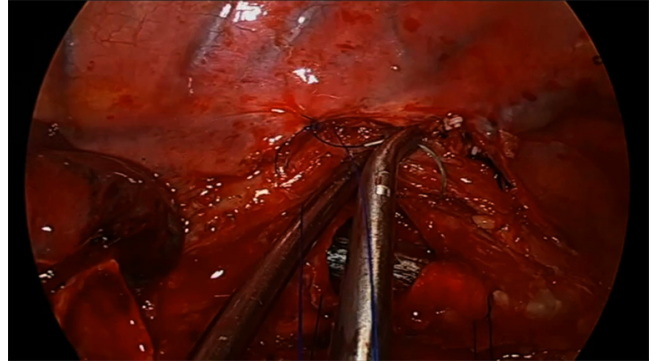

Figure 6 Advancement of the endotracheal tube into the LMB after completion of the running suture of the posterior and lateral wall between the trachea and LMB. LMB, left main bronchus.

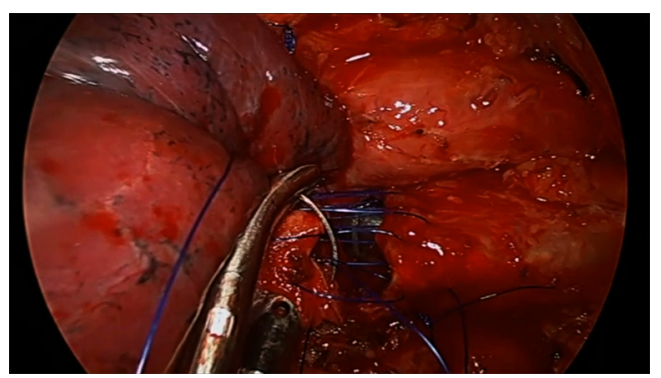

Figure 7 Completion of the anastomosis for carinal reconstruction.

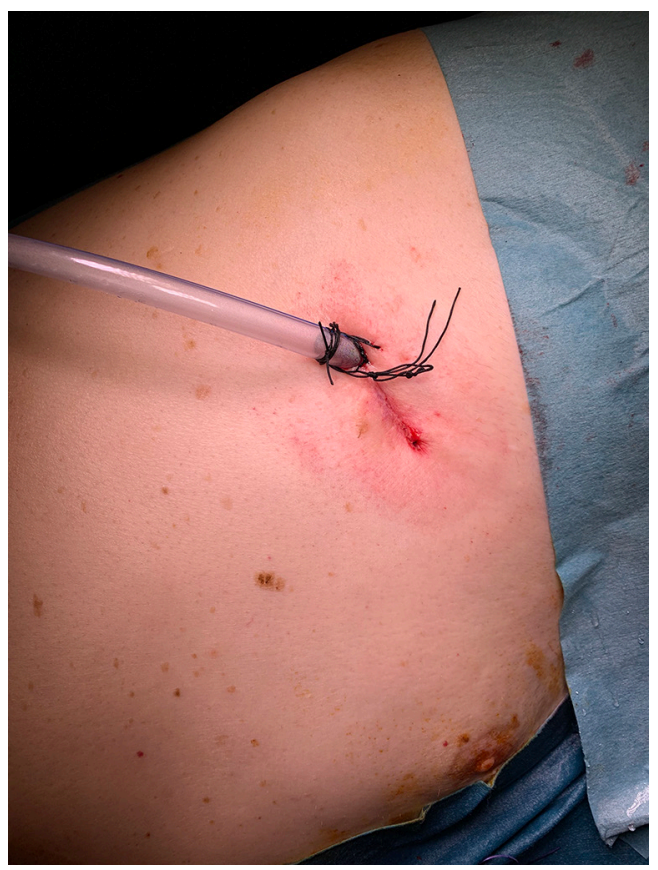

Figure 8 One chest tube is place at the incision after completion of the procedure.

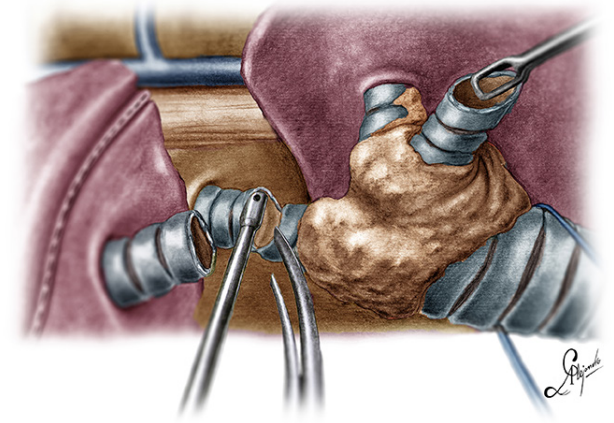

Figure 9 Carinal resection in-block with the RUL because of tumor extension into RUB. RUL, right upper lobe; RUB, right upper bronchus.

needle $3 / 0$ polipropilene, the suture is started outside-in between the junction of the membranous and cartilaginous part of the trachea and the same junction of the LMB and into the lateral wall of the LMB, until completion of half of the circumference of the trachea and the LMB (Figure 6). With the second needle, a running suture is continued between the membranous part of the trachea and LMB. Afterwards, with a separate suture a neocarina is form by suturing the medial wall of the LMB to the medial wall of the right main bronchus (RMB). This suture is tied to the suture connecting the anterior wall of the trachea and RMB. Finally the rest of the anterior and lateral wall of the $\mathrm{RMB}$ and $\mathrm{LMB}$ can be anastomosed to the trachea (Figure 7) $(40,43)$. After completion of the procedure, usually one chest tube placed at the same incision is left (Figure 8).

One of the most difficult scenarios is when a right upper lobe tumor is extending into the carina since a carinal resection with a right upper lobectomy has to be perform in order to make an in-block resection of the tumor (Figure 9). The reconstruction is especially difficult since there is a considerable mismatch between the diameter of the intermedius bronchus to the distal trachea and LMB. There are two options for reconstruction by uniportal VATS, the first is following the same steps as lung sparing carinal resection, starting with the lateral wall of the LMB to the distal trachea, following by the formation of the neocarina between the LMB and the intermediate bronchus and reimplantation of the neocarina to the trachea. The second option is to perform a complete end to end anastomosis between de trachea and the LMB and reimplantation on the intermediate bronchus to the lateral cartilaginous wall of the 


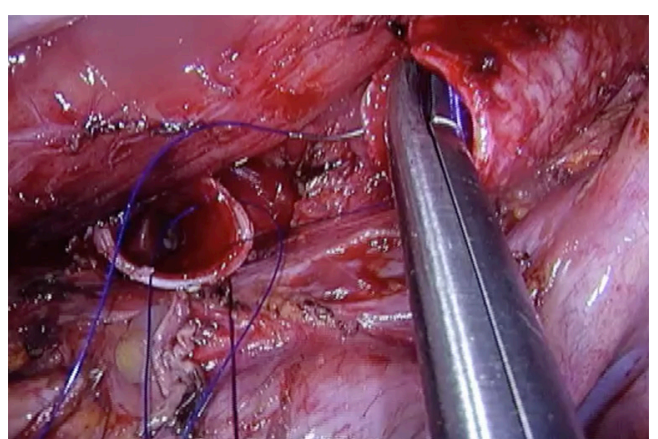

Figure 10 Running suture between the trachea and LMB after right sleeve pneumonectomy. LMB, left main bronchus.

trachea, usually at least $2 \mathrm{~cm}$ above the first anastomosis (40).

A sleeve pneumonectomy is performed when the extension of the tumor into or from the lung precludes a lung sparing procedure. For a right sleeve pneumonectomy, the incision is placed in the mid-axillary line, dissection of the hilar structures is performed and dissection and exposure of the trachea, RMB and LMB is carried out. After stapling and division of the vascular structures, the $\mathrm{RMB}$ is transected close to the carina and the specimen is removed. The anastomosis is performed in an end-to-end fashion between the trachea and the LMB (Figure 10). The suture is began first suturing the left side wall of the trachea to the left side wall of the LMB, then membranous portion and, finally, right side of trachea and LMB. During the anastomosis, oxygenation is also usually maintained with the help of HFJV catheter, facilitating the running suture with minimal interference (Figure 11).

Left sleeve pneumonectomy is a very rare procedure because the LMB is quite longer and tumors extending into de carina from this side usually involve the sub aortic space structures. The preferred approach is via median sternotomy, because it provides good exposure of the carina and avoids a complex anastomosis behind the aortic arch (9).

The first reported cases of carinal and tracheal resections in the 90 's, had mortality rates as high as $20.9 \%$ (44). Even in 2000 decade, the rate of complications was reported with a wide variability, with a top value of $50.8 \%$ (45). In recent years and in centers with experienced surgeons, and with the advent of minimally invasive approaches, the mortality rate is lower with overall postoperative 30-day rates between $2.7 \%$ to $10.9 \%$, but still with a wide range of morbidity $(12,46,47)$. Also, reports of new techniques like carinal resections with lung parenchyma-sparing for NSCLC by uniportal VATS give a new perspective to this

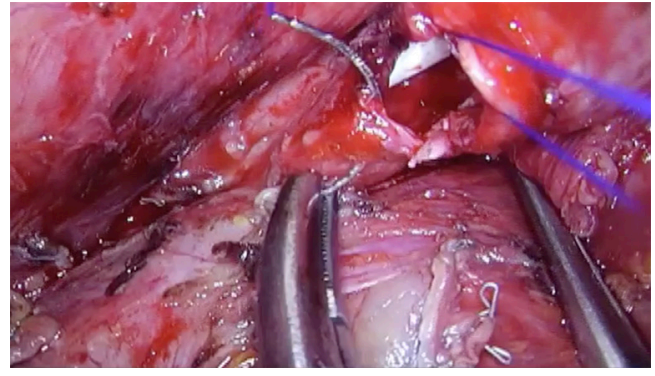

Figure 11 Completion of the anastomosis after right carinal pneumonectomy with the aid of HFJV. HFJV, high frequency jet ventilation.

kind of complex cases.

\section{Complications of carinal and tracheal resections}

Prevention of complications is the most important factor in the management of this patients. An aggressive approach must be implemented as soon as possible if a complication arises (48).

In the postoperative period, the patient must be preferably extubated in the operating room to avoid positive pressure ventilation in the new airway anastomosis. With an enhanced recovery after surgery protocol (ERAS) implemented with early mobilization, good pain control and respiratory chest physiotherapy, atelectasis, and pneumonia secondary to mucocilliary clearance disruption can be avoided $(6,10,49)$.

After a carinal or tracheal resection surgery, some authors recommended neck flexion at least for 7 days, using a stich between the pre sternal skin and the chin to prevent tension in the anastomosis (50), but nowadays, the use of the stitch is not mandatory and it can result in unnecessary pain for the patient $(6,10)$.

The mortality rate for carinal resection is $3-20 \%$ with an overall morbidity of $11-50 \%(6,10,48,50)$. Early mortality is related to nosocomial pneumonia and ARDS, besides late mortality is more related to anastomotic complications $(48,51)$.

ARDS is a high mortality complication especially after carinal resections with sleeve pneumonectomy (52). Postpneumonectomy pulmonary edema has $50-100 \%$ of mortality risk, with an acute onset of tachypnea, hypoxia, hypercapnia and ground-glass infiltrates on chest imaging. It can be seen in $4-14 \%$ of carinal resections, and is more associated to ventilator-induced barotrauma, fluid overload, blood transfusion in the perioperative period 
and micro aspirations. The treatment is only supportive, with an adequate mechanical ventilation, use of antibiotics, avoid excess of intravenous fluids, pulmonary toilet, and sometimes, the use of ECMO $(6,48,52)$.

Atrial fibrillation is the most common cardiac arrhythmia in these patients. Control of heart rate and rhythm is mandatory and can be achieved with beta-blockers, amiodarone, and calcium blockers (53). If the patient is unstable, electrical synchronic cardioversion must be done early to revert the abnormal rhythm. Anticoagulation can be started only when there is no risk of bleeding $(10,54)$.

Anastomotic complications can vary in presentation, from granulation tissue formation, various degrees of mucosal necrosis, dehiscence of anastomosis with a bronchopleural fistula to life-threaten bronchovascular fistula $(6,55)$.

Dehiscence of the anastomosis is the most feared complication in these complex procedures, it can lead to mediastinitis and loss of the patent airway. Stridor, wound infection and respiratory distress are usually the presenting symptoms. Bronchoscopy must be performed as early as possible to confirm diagnosis; and then, a personalized management must be required to repair the anastomosis. In some cases, the use of an airway stent to cover the defect can be an option, but an early intervention should be the rule (56).

Bronchopleural fistulas have an incidence of $3.8 \%$ to $21.6 \%$ and more than $8 \%$ for sleeve resections $(48,57,58)$. This complication can be classified according to time of onset after the procedure in late (more than 30 days), intermediate (8-30 days) and early (1-7 days) (45). Early bronchopleural fistulas usually present as large air leaks with progressive subcutaneous emphysema, dyspnea, and hemodynamic instability. Clinical manifestations of late onset are productive cough, fever, and an air-fluid level on chest imaging (48).

The most important risk factors for developing a bronchopleural fistula are ischemia and tension at the anastomosis $(7,59)$. To reduce the risk, it is very important to preserve the bronchial and carinal vascularization, perform a tension-free anastomosis and cover the suture with a well-vascularized tissue $(16,58)$, with no difference between running and interrupted suture technique (60). Some authors have found that wrapping of the anastomosis can be avoided if all the peri bronchial tissue is preserved as much as possible without adding more complications to the procedure (61), even if the patient has received neoadjuvant chemo- or chemoradiotherapy (62).

Early bronchopleural fistula is amenable to single stage repair, debridement, and removal of necrotic tissue, using a patch of well vascularized tissue to buttress the repair (intercostal muscle, diaphragm, omentum) (63). If it is not feasible to do a primary repair, a pneumonectomy should be performed. The pleural space must be evacuated in an effective way, using of broad-spectrum antibiotics and reviewing the anastomotic defect. A persistent contaminated pleural space can be managed with continuous drainage with an open thoracostomy, a thoracoplasty and the use of muscles flaps to obliterate the pleural space $(48,57)$.

A bronchovascular fistula occurs in less than $3 \%$ of carinal resections, but it has a high mortality rate secondary to massive hemoptysis and respiratory distress (64). Bronchoscopy must be performed immediately and the patient must undergo surgery if confirmed (48).

Benign anastomotic strictures or stenosis can occur secondary to granulation tissue formation in $2.5-7.4 \%$ of patients after carinal resection and is more frequent with the use of non-absorbable sutures with scars due to local isquemia. The patient may present post obstructive pneumonitis, pneumonia, dyspnea, stridor, cough or wheezing from days to weeks after surgery. The diagnosis can be confirmed with flexible bronchoscopy, and in the same interventional time, can be resolved with rigid bronchoscopy, endoscopic dilation, endoscopic laser or an airway stent. If the stricture or obstruction is severe, or the patient has repeated infections or collapse of the remaining lung without an effective endoscopic treatment, a segmental resection of the stricture and re-anastomosis usually is very difficult and dangerous because of scar tissue and adhesions. Sometimes a pneumonectomy is indicated as a definitive surgical treatment (48).

A local recurrence at the anastomosis is a late complication of tracheal and carinal resections, and it can be seen in less than $5-10 \%$ of carinal and sleeve resections. The malignant involvement of margins is the most important risk factor for local recurrence. If the patient is not suitable for pneumonectomy, radiotherapy and the use of interventional bronchoscopy is a good option to palliate symptoms (6).

\section{Is there a change in the approach for distal tracheal and carinal NSCLC in our era?}

The evolution of oncologic thoracic surgery has been taking giant leaps over the last decade in minimally invasive surgery. There has been a lot of progress in the morbimortality of tracheal and carinal resections for NSCLC 
in a way that has shifted the discussion is it feasible to perform this procedure with good oncological results to which is the best minimal invasive approach to operate on these patients. The limits are continuously pushed and already some groups are successfully carrying out complex carinal reconstructions by uniportal VATS in nonintubated patients, a scenario that a few years ago would have been considered impossible. Experience is scarce and the advantages of this approaches are still in debate since, for the moment, only few groups are able to perform this kind of highly complex procedures. However, as experience grows, feasibility will be mainly related to the experience of the surgeon and anesthesiologist in charge of such cases. As recommended for other complex cases, these surgeries should only be performed by highly experience teams with minimally invasive airway reconstruction surgery in high volume referral centers.

\section{Acknowledgments}

Funding: None.

\section{Footnote}

Provenance and Peer Review: This article was commissioned by the Guest Editors (Davide Tosi and Alessandro Palleschi) for the series "The Treatment of Locally Advanced Lung Cancer" published in Current Challenges in Thoracic Surgery. The article has undergone external peer review.

Conflicts of Interest: All authors have completed the ICMJE uniform disclosure form (available at https://ccts. amegroups.com/article/view/10.21037/ccts-20-95/coif). The series "The Treatment of Locally Advanced Lung Cancer" was commissioned by the editorial office without any funding or sponsorship. Dr. González-Rivas serves as an unpaid editorial board member of Current Challenges in Thoracic Surgery from January 2020 to December 2021. The authors have no other conflicts of interest to declare.

Ethical Statement: The authors are accountable for all aspects of this work in ensuring that questions related to the accuracy or integrity of any part of this work are appropriately investigated and resolved.

Open Access Statement: This is an Open Access article distributed in accordance with the Creative Commons Attribution-NonCommercial-NoDerivs 4.0 International
License (CC BY-NC-ND 4.0), which permits the noncommercial replication and distribution of the article with the strict proviso that no changes or edits are made and the original work is properly cited (including links to both the formal publication through the relevant DOI and the license). See: https://creativecommons.org/licenses/by-nc-nd/4.0/.

\section{References}

1. Abbott OA. Experiences with the surgical resection of the human carina, tracheal wall, and contralateral bronchial wall in cases of right total pneumonectomy. J Thorac Surg 1950;19:906-22.

2. Barclay RS, McSwan N, Welsh TM. Tracheal reconstruction without the use of grafts. Thorax 1957;12:177-80.

3. Gonzalez-Rivas D. Uniportal thoracoscopic surgery: from medical thoracoscopy to non-intubated uniportal videoassisted major pulmonary resections. Ann Cardiothorac Surg 2016;5:85-91.

4. Guido-Guerrero W, Bolaños-Cubillo A, GonzálezRivas D. Single-port video-assisted thoracic surgery (VATS)—advanced procedures \& update. J Thorac Dis 2018;10:S1652-61.

5. Stevic R, Milenkovic B. Tracheobronchial tumors. J Thorac Dis 2016;8:3401-13.

6. Weder W, Inci I. Carinal resection and sleeve pneumonectomy. J Thorac Dis 2016;8:S882-8.

7. Tapias L, Lanuti M. Carinal resections. Ann Cardiothorac Surg 2018;7:309-13.

8. Madariaga MLL, Gaissert HA. Overview of malignant tracheal tumors. Ann Cardiothorac Surg 2018;7:244-54.

9. Deslauriers J, Grégoire J, Jacques LF, et al. Sleeve pneumonectomy. Thorac Surg Clin 2004;14:183-90.

10. Petrov RV, Bakhos CT, Abbas AE. Carinal resection. Shanghai Chest Shanghai Chest. 2018;2:84.

11. Galetta D, Spaggiari L. Early and Long-Term Results of Tracheal Sleeve Pneumonectomy for Lung Cancer After Induction Therapy. Ann Thorac Surg 2018;105:1017-23.

12. Sezen CB, Kocaturk CI, Bilen S, et al. Long-Term Outcomes of Carinal Sleeve Resection in Non-Small Cell Lung Cancer. Thorac Cardiovasc Surg 2020;68:190-8.

13. Yang $Z$, Zhai C. Uniportal video-assisted thoracoscopic surgery following neoadjuvant chemotherapy for locallyadvanced lung cancer. J Cardiothorac Surg 2018;13:33.

14. Pfannschmidt J, Kollmeier J. [Results of N1 and N2 surgery in non-small cell lung cancer]. Chirurg 2019;90:974-81. 
15. Mehran R, Deslauriers J. Carinal Pneumonectomy. Thorac Surg Clin 2018;28:315-22.

16. Orlowski TM, Dziedzic D. Carinal Resection and Reconstruction. Thorac Surg Clin 2018;28:305-13.

17. Mathisen D. Distal Tracheal Resection and Reconstruction: State of the Art and Lessons Learned. Thorac Surg Clin 2018;28:199-210.

18. Brunelli A, Kim AW, Berger KI, et al. Physiologic evaluation of the patient with lung cancer being considered for resectional surgery: Diagnosis and management of lung cancer, 3rd ed: American College of Chest Physicians evidence-based clinical practice guidelines. Chest 2013;143:e166S-90S.

19. Della Rocca G, Vetrugno L, Coccia C, et al. Preoperative Evaluation of Patients Undergoing Lung Resection Surgery: Defining the Role of the Anesthesiologist on a Multidisciplinary Team. J Cardiothorac Vasc Anesth 2016;30:530-8.

20. Salati M, Brunelli A. Risk Stratification in Lung Resection. Curr Surg Rep 2016;4:37.

21. Spyratos D, Zarogoulidis P, Porpodis K, et al. Preoperative evaluation for lung cancer resection. J Thorac Dis 2014;6 Suppl 1:S162-6.

22. Chitilian HV, Bao X, Mathisen DJ, et al. Anesthesia for Airway Surgery. Thorac Surg Clin 2018;28:249-55.

23. Smeltz AM, Bhatia M, Arora H, et al. Anesthesia for Resection and Reconstruction of the Trachea and Carina. J Cardiothorac Vasc Anesth 2020;34:1902-13.

24. Hoetzenecker K, Klepetko W, Keshavjee S, et al. Extracorporeal support in airway surgery. J Thorac Dis 2017;9:2108-17.

25. McRae K, de Perrot M. Principles and indications of extracorporeal life support in general thoracic surgery. J Thorac Dis 2018;10:S931-46.

26. Ujiie H, Yasufuku K. New era of "resection of the carina and lower trachea." J Thorac Dis 2017;9:4932-6.

27. Liang H, Gonzalez-Rivas D, Zhou Y, et al. Nonintubated Anesthesia for Tracheal/Carinal Resection and Reconstruction. Thorac Surg Clin 2020;30:83-90.

28. Zuin A, Mammana M, Rea F. Non-intubated tracheal surgery: is it worthwhile? J Thorac Dis 2017;9:3663-6.

29. Jiang L, Liu J, Gonzalez-Rivas D, et al. Thoracoscopic surgery for tracheal and carinal resection and reconstruction under spontaneous ventilation. J Thorac Cardiovasc Surg 2018;155:2746-54.

30. Nakanishi K, Kuruma T. Video-assisted thoracic tracheoplasty for adenoid cystic carcinoma of the mediastinal trachea. Surgery 2005;137:250-2.
31. Nakanishi R, Yamashita T, Muranaka K, et al. Thoracoscopic carinal resection and reconstruction in a patient with mucoepidermoid carcinoma. J Thorac Cardiovasc Surg 2013;145:1134-5.

32. Li J, Wang W, Jiang L, et al. Video-Assisted Thoracic Surgery Resection and Reconstruction of Carina and Trachea for Malignant or Benign Disease in 12 Patients: Three Centers' Experience in China. Ann Thorac Surg 2016;102:295-303.

33. Xu X, Chen H, Yin W, et al. Thoracoscopic half carina resection and bronchial sleeve resection for central lung cancer. Surg Innov 2014;21:481-6.

34. Jiao W, Zhu D, Cheng Z, et al. Thoracoscopic tracheal resection and reconstruction for adenoid cystic carcinoma. Ann Thorac Surg 2015;99:e15-17.

35. Lin J, Kang M, Chen S, et al. Video-assisted thoracoscopic right upper lobe sleeve lobectomy combined with carinal resection and reconstruction. J Thorac Dis 2015;7:1861-4.

36. Lyscov A, Obukhova T, Ryabova V, et al. Double-sleeve and carinal resections using the uniportal VATS technique: a single centre experience. J Thorac Dis 2016;8:S235-41.

37. Gonzalez-Rivas D, Yang Y, Sekhniaidze D, et al. Uniportal video-assisted thoracoscopic bronchoplastic and carinal sleeve procedures. J Thorac Dis 2016;8:S210-22.

38. Qiu T, Zhao Y, Song J, et al. Two-port approached thoracoscopic carina reconstruction using natural bronchial bifurcation. J Cardiothorac Surg 2016;11:147.

39. Yang C, Abu Akar F, Chen J, et al. Right sleeve pneumonectomy via uniportal video-assisted thoracoscopic approach. J Thorac Dis 2018;10:E391-6.

40. Gonzalez-Rivas D, Yang Y, Stupnik T, et al. Uniportal video-assisted thoracoscopic bronchovascular, tracheal and carinal sleeve resections $†$. Eur J Cardiothorac Surg 2016;49 Suppl 1:i6-16.

41. Gonzalez-Rivas D. VATS lobectomy: surgical evolution from conventional VATS to uniportal approach. ScientificWorldJournal 2012;2012:780842.

42. Liu Z, Yang R, Shao F. Comparison of Postoperative Pain and Recovery between Single-Port and Two-Port Thoracoscopic Lobectomy for Lung Cancer. Thorac Cardiovasc Surg 2019;67:142-6.

43. Gonzalez-Rivas D, Soultanis KM, Garcia A, et al. Uniportal video-assisted thoracoscopic lung sparing tracheo-bronchial and carinal sleeve resections. J Thorac Dis [Epub ahead of print].

44. Tedder M, Anstadt MP, Tedder SD, et al. Current morbidity, mortality, and survival after bronchoplastic procedures for malignancy. Ann Thorac Surg 
1992;54:387-91.

45. Regnard J-F, Perrotin C, Giovannetti R, et al. Resection for tumors with carinal involvement: technical aspects, results, and prognostic factors. Ann Thorac Surg 2005;80:1841-6.

46. Costantino CL, Geller AD, Wright CD, et al. Carinal surgery: A single-institution experience spanning 2 decades. J Thorac Cardiovasc Surg 2019;157:2073-83.e1.

47. Peng Q, Zhang L, Ren Y, et al. Reconstruction of Long Noncircumferential Tracheal or Carinal Resections With Bronchial Flaps. Ann Thorac Surg 2019;108:417-23.

48. Tapias LF, Ott HC, Mathisen DJ. Complications Following Carinal Resections and Sleeve Resections. Thorac Surg Clin 2015;25:435-47.

49. Batchelor TJP, Rasburn NJ, Abdelnour-Berchtold E, et al. Guidelines for enhanced recovery after lung surgery: recommendations of the Enhanced Recovery After Surgery (ERAS®) Society and the European Society of Thoracic Surgeons (ESTS). Eur J Cardiothorac Surg 2019;55:91-115.

50. Yildizeli B, Fadel E, Mussot S, et al. Morbidity, mortality, and long-term survival after sleeve lobectomy for non-small cell lung cancer. Eur J Cardiothorac Surg 2007;31:95-102.

51. Casiraghi M, Mariolo AV, Galetta D, et al. Carinal resection: technical tips. J Vis Surg 2018;4:122.

52. Kometani T, Okamoto T, Yoshida S, et al. Acute respiratory distress syndrome after pulmonary resection. Gen Thorac Cardiovasc Surg 2013;61:504-12.

53. Roselli EE, Murthy SC, Rice TW, et al. Atrial fibrillation complicating lung cancer resection. J Thorac Cardiovasc Surg 2005;130:438-44.

54. Onaitis M, D'Amico T, Zhao Y, et al. Risk factors for atrial fibrillation after lung cancer surgery: analysis of the Society of Thoracic Surgeons general thoracic surgery database. Ann Thorac Surg 2010;90:368-74.

doi: $10.21037 /$ ccts-20-95

Cite this article as: Guido-Guerrero W, Bolaños-Cubillo A, García-Pérez A, González-Rivas D. Tracheal and carinal resections in the treatment of non-small cell lung cancer: a trend to a minimally invasive approach. Curr Chall Thorac Surg 2021;3:27.
55. Bylicki O, Vandemoortele T, Orsini B, et al. Incidence and management of anastomotic complications after bronchial resection: a retrospective study. Ann Thorac Surg 2014;98:1961-7.

56. Auchincloss HG, Wright CD. Complications after tracheal resection and reconstruction: prevention and treatment. J Thorac Dis 2016;8:S160-7.

57. Ludwig C. [Surgical treatment of tracheopleural and bronchopleural fistulas after bronchoplastic resection (sleeve or bifurcation resection)]. Chirurg 2019;90:704-9.

58. Luciano G, Stella F, Dell'Amore A, et al. Tracheobronchoplastic procedures for NSCLC: single-centre experience. Heart Lung Circ 2015;24:1027-32.

59. Farkas EA, Detterbeck FC. Airway complications after pulmonary resection. Thorac Surg Clin 2006;16:243-51.

60. Palade E, Holdt H, Passlick B. Bronchus anastomosis after sleeve resection for lung cancer: does the suture technique have an impact on postoperative complication rate? Interact Cardiovasc Thorac Surg 2015;20:798-804.

61. Konstantinou M, Potaris K, Sakellaridis T, et al. Sleeve lobectomy for patients with non-small-cell lung cancer: a simplified approach. Eur J Cardiothorac Surg 2009;36:1045-9.

62. Storelli E, Tutic M, Kestenholz P, et al. Sleeve resections with unprotected bronchial anastomoses are safe even after neoadjuvant therapy. Eur J Cardiothorac Surg 2012;42:77-81.

63. Shrager JB, Wain JC, Wright CD, et al. Omentum is highly effective in the management of complex cardiothoracic surgical problems. J Thorac Cardiovasc Surg 2003;125:526-32.

64. Conlan AA, Hurwitz SS, Krige L, et al. Massive hemoptysis. Review of 123 cases. J Thorac Cardiovasc Surg 1983;85:120-4. 